\title{
Reconstruction of the Areola-Nipple Complex after Mastectomy
}

\author{
Tamayo Carbon AM1, Vila Garcia E2* and Cedillo Lopez RM³ \\ ${ }^{1}$ Specialists of II degree in Plastic Surgery and Caumatology, Surgical Hospital \\ Hermanos Ameijeiras, Cuba \\ ${ }^{2}$ Specialist I Degree in General Surgery, Surgical Hospital Hermanos Ameijeiras, \\ Cuba
}

Conceptual Paper

Volume 3 Issue 1

Received Date: April 02, 2019

Published Date: April 17, 2019

DOI: $10.23880 /$ ijtps-16000129

${ }^{3}$ Specialists of I degree in Plastic Surgery and Caumatology, Surgical Hospital Hermanos Ameijeiras, Cuba

*Corresponding author: Eyleen Vila Garcia, Assistant Professor, Services of Plastic Surgery and Caumatology and General Surgery, Surgical Hospital Hermanos Ameijeiras, Havana City, Cuba, Tel: 53232327; Email: hielenvg@infomed.sld.cu

\section{Abstract}

Introduction: The creation of the Areola-Nipple complex is the last time of breast reconstruction, transforming the breast mound into a real breast.

Objective: Show the results, advantages and disadvantages of different techniques applied in the reconstruction of the Areola-Nipple Complex.

Material and methods: A retrospective descriptive study of 70 patients with oncological deformities of the breast at Hermanos Ameijeiras Hospital from January 2008 to December 2018 was carried out.

Results: 18 patients were created the nipple by local flaps method, 44 were grafted and 8 without reconstruction since it was not desired, and others had no projection of the healthy nipple. Of these 53 were tattooed, 15 grafted with contralateral areola and 2 without reconstruction. There were no complications in the contralateral nipple graft, but with the other method $100 \%$ suffered flattening with regular results. Of the 53 tattooed patients, good results predominated and only 16 had slight pigmentation disorders.

Conclusions: The reconstruction of the Areola-Nipple Complex after ablative surgery of the breast transforms the breast of more natural aspect and the ideal methods are the contralateral nipple graft and the intradermal tattoo.

Keywords: Areola-Nipple Complex; Contralateral nipple; Intradermal tattoo

\section{Introduction}

The formation of the Areola-Nipple Complex (ANC) corresponds to the final part of the reconstruction of the breast mound. There are different techniques that allow to improve the anatomical and aesthetic appearance of the breast; in addition to improving biopsychosocial conditions, mastectomized women [1]. 


\section{International Journal of Transplantation \& Plastic Surgery}

There are different surgical techniques described in the medical literature to reconstruct the areola-nipple complex, most of them easy to reproduce, but difficult to learn, with variable aesthetic results, according to the experience of the surgeon [2].

In the reconstruction of the ANC it is necessary to establish two objectives:

1) Reconstruction of the nipple (teloplasty): A structure is created that projects similarly in volume and position to the contralateral nipple.

2) Reconstruction of the areola: With a pigmented area of configuration, location and color, most similar to the contralateral areola.

The reconstruction of the ANC aims to achieve an adequate symmetry in: position, size, shape, texture, pigmentation and permanent projection of the nipple. To achieve this it is advisable to defer it until the reconstructed breast has reached its final shape and position; in addition to the greater degree of symmetry with respect to the contralateral breast, around 3 to 6 months $[3,4]$.

It represents the simplest from a technical perspective, but it is among the most important from an aesthetic perspective. Studies have shown that ANC reconstruction has a high correlation with patient's overall satisfaction and acceptance of body image. The completion of breast reconstruction with the creation of a ANC that coincides with the contralateral nipple (in terms of size, shape, projection and position) is significantly added to the reconstructive results $[1,5]$.

There are multiples reconstruction techniques but none has been so successful as to become the procedure of choice. They are grouped into 2 groups: local flaps and autologous grafts. The first ones based on the creation of prominence from neighborhood flaps with random flow, the main problem being the loss of projection. The viability of these flaps can be affected by radiotherapy and in breasts with multiples scars derived from successive surgeries that compromise the vascular flow of the flaps $[4,6,7]$.

The areolar reconstruction also has two ways to perform: autografts and tattoos. Of the former, the contralateral areola is ideal for pigmentation, especially when the healthy breast requires symmetry. Sophistication has been described in the literature that includes repairing the Montgomery tubers with graft composed of skin and cartilage taken from the auricular shell with the particularity that there is no optimal relationship between the benefits obtained and the tissue sacrifice that takes place in the area donor. With the tattoo technique a new areola of color can be produced very similar to the contralateral $[4,5]$.

\section{Material and Methods}

Patients treated in the protocol consultation of oncological deformities of the breast of the Hermanos Ameijeiras Hospital in the period from January 2008 to December 2018. This reconstructive phase of the ANC was carried out in a deferred manner, developed in a single surgical time, with local anesthesia and on an outpatient basis, without hospital admission.

The results were grouped in:

- Good: 50 of the symmetry criteria are met.

- Regular: Only 20 of the symmetry criteria are met; Some complication occurred or the nipple flattened. - Bad: Total failure.

\section{Results}

Of the 70 patients with culmination of the surgical times to 18 were created nipple by the method of local flaps, 44 were grafted and 8 without reconstruction because it was not desired, and others had no projection of the healthy nipple. Of these 53 were tattooed, 15 grafted with contralateral areola and 2 without reconstruction. The postoperative behavior of one year did not show complications in the contralateral nipple graft while the other method showed partial necrosis, dehiscence, hypertrophic scars and worse, $100 \%$ suffered flattening, this technique being responsible for the regular results. Of the 53 tattooed patients only 16 had slight pigmentation disorders due to the difficulty in acquiring the pigments and not the procedure itself that offers excellent results in terms of color. The grafts presented a partial necrosis and two unsightly scars.

Good results prevailed in the present study, but not significantly, as there was a similar number of patients reconstructed with the two nipple techniques and the 15 cases of local flaps were flattened. No failure was collected (Figures 1-3). 


\section{International Journal of Transplantation \& Plastic Surgery}
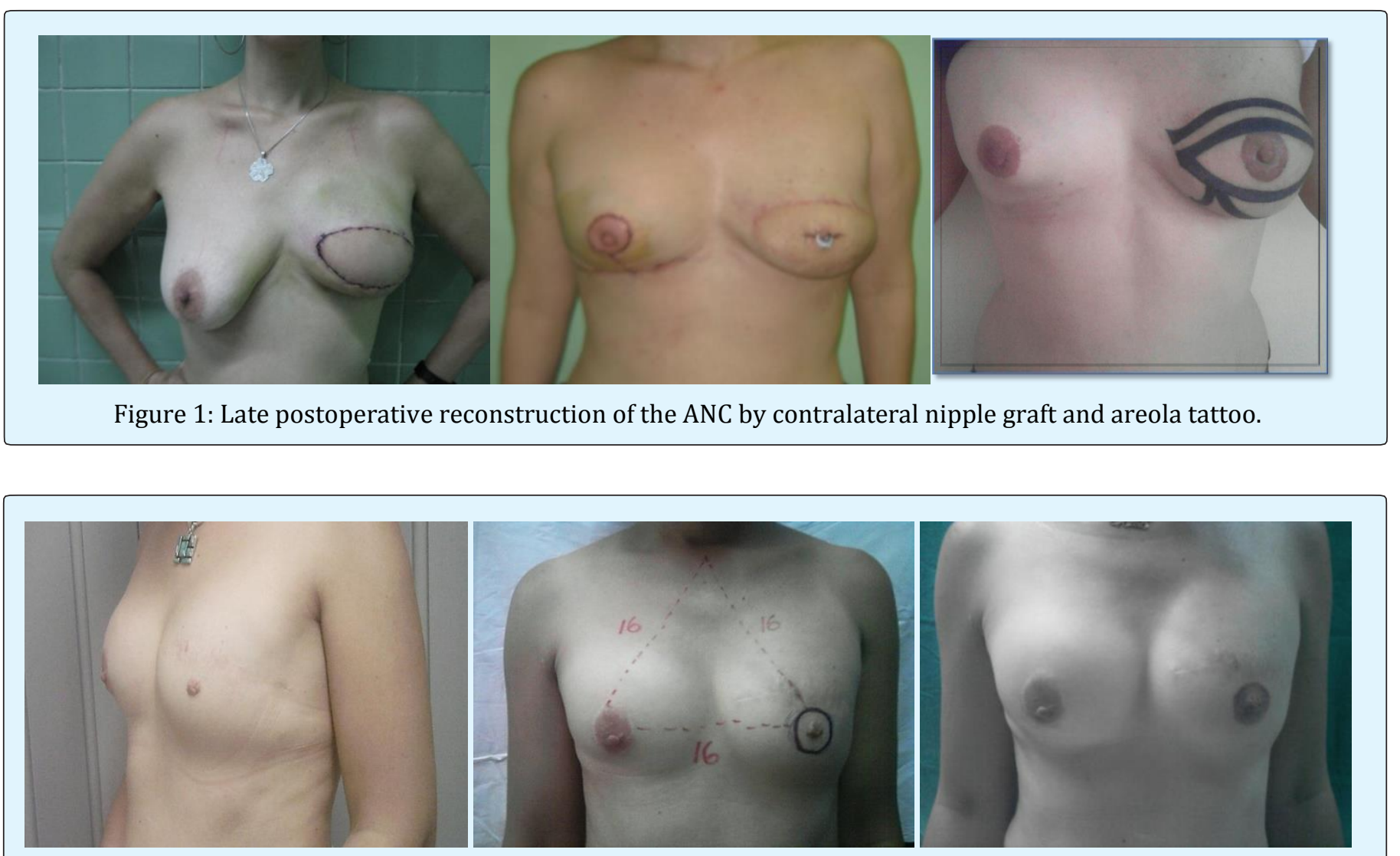

Figure 2: Late postoperative reconstruction of the ANC by contralateral nipple graft and areola tattoo.
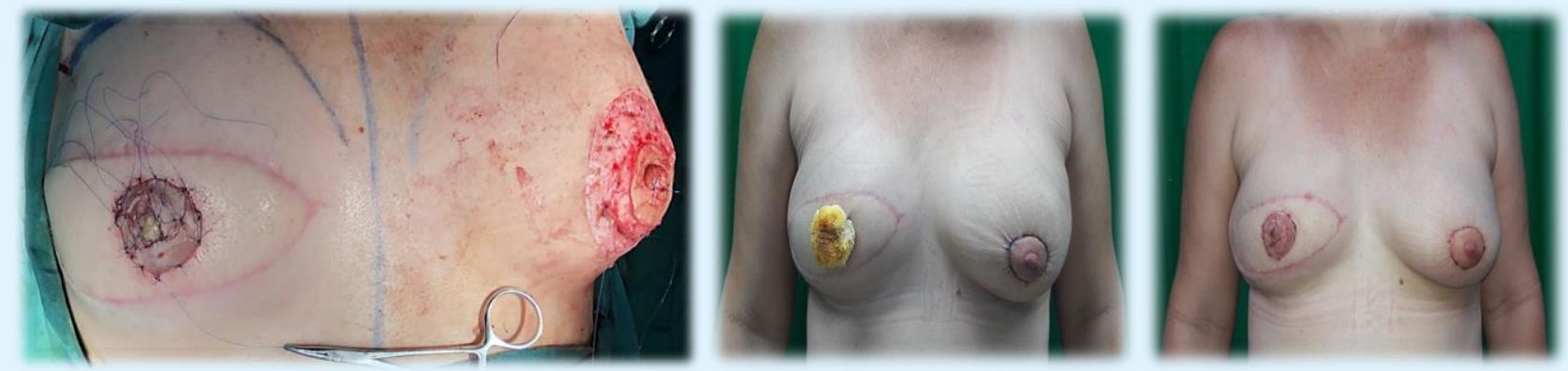

Figure 3: ANC reconstruction with contralateral nipple and areola graft.

\section{Discussion}

Sisti, et al. [2] performed a review of the literature on the techniques for ANC reconstruction of 75 articles between 1946 and 2015. The complications in the reconstruction of the nipple were $46.9 \%$ after grafting, $7.9 \%$ after the flap local and $5.3 \%$ in the case of flaps with autologous / alloplastic graft / allograft, while complications in the Areola reconstruction were $10.1 \%$ after grafting and $1.6 \%$ after tattooing. The loss of projection, although considerable (45\%-75\%), did not have a significant impact on patient satisfaction. In our study, the grafted nipples did not present complications, unlike those reconstructed with local random flow flaps 


\section{International Journal of Transplantation \& Plastic Surgery}

that were mostly flattened, since only one technique was used for the projection, being the ideal anatomical projection of the nipple must be greater or equal to $1 \mathrm{~cm}$ [12].

However, there are other techniques to give projection as is the case of the tetris-type flap [9], which is a local flap that is easy to reproduce and has good aesthetic results. The greater projection it offers is directly related to the donor skin of the reconstruction of the breast mound. The average 6-month projection is $8 \mathrm{~mm}$.

Another type of techniques such as the skate flap or the star flap, report projection ranges from 5 to $9.2 \mathrm{~mm}$, Sisti [2] mentions that the projection loss margins are up to $50 \%$. Jalini [10] describes the creation of the nipple with the C-V flap; Zhong [11] performed an ambispective study in which he evaluated the modified skate technique for nipple reconstruction.

Currently, the boomerang type flap described by Kim suggests the largest projection at 14 months above 10 $\mathrm{mm}$; however, in this type of flap whose structure is formed by the scar of previous reconstruction of the breast, it is likely to present a high risk of necrosis due to the microcirculation being affected [13].

The tattoo is the other main complement of the areola reconstruction. Whether worn alone or in conjunction with skin grafts, tattoos can provide excellent areolar combination of colors with limited morbidity. Initially introduced by Rees and Spear in 1975 [1]. Sisti, et al. [2] found in the review of literature the tattoo as safer than the graft in the reconstruction of the areola.

In tattooing in unilateral cases, slightly more pigmented colors should be chosen than the contralateral areola. Lanza and Arias found that $9.5 \%$ of the areolas needed retouching to pigment fading and it is likely that he will need to retouch the tattoo after several months or years to achieve an aesthetically symmetric color match [7-9,14].

\section{Conclusions}

The reconstruction of CAP after ablative surgery of the breast is the last time of postmastectomy breast reconstruction and is included in the comprehensive treatment of breast cancer. It allows the woman to see her whole body again by transforming the breast mound into a real breast. The ideal method is the contralateral nipple graft and intradermal tattoo.

\section{References}

1. Bykowski MR, Emelife PI, Emelife NN, Chen W, Panetta NJ, et al. (2017) Nipple-areola complex reconstruction improves psychosocial and sexual well-being in women treated for breast cancer. J Plast Reconstr Aesthet Surg 70(2): 209-214.

2. Sisti A, Grimaldi L, Tassinari J, Cuomo R, Fortezza L, et al. (2016) Nipple-areola complex reconstruction techniques: A literature review. Eur J Surg Oncol 42(4): 441-465.

3. Nimboriboonporn A, Chuthapisith (2014) Nippleareola complex reconstruction. Gland Surgery 3(1): $35-42$.

4. Henderson JT, Lee TJ, Swiergosz AM, Hiller AR, Choo J, et al. (2018) Nipple-Areolar Complex Reconstruction: A Review of the Literature and Introduction of the Rectangle-to-Cube Nipple Flap. Eplasty 18: e15.

5. American Society of Plastic Surgeons (2018) Breast Reconstruction.

6. Momoh AO, Colakoglu S, de Blacam C, Yueh JH, Lin SJ, et al. (2012) The impact of nipple reconstruction on patient satisfaction in breast reconstruction. Ann Plast Surg 69(4): 389-393.

7. Highton LR, Murphy JA (2017) Immediate NippleAreolar Complex Reconstruction for Patients Undergoing Implant-Based Reconstruction or Therapeutic Mammoplasty. Plast Reconstr Surg Glob Open 5(9): e1243.

8. Tomita S, Mori K, Miyawaki T (2018) Color Change After Paramedical Pigmentation of the Nipple-Areola Complex. Aesthetic Plast Surg 42(3): 656-661.

9. Fernandez Garcia R, Fernandez Delgado J, Martinez Méndez JR, Bravo Brañas E, , García Redondo M, et al. (2017) Areola-nipple complex reconstrution. Iberolatinoam Plastic Surgery 33(2): 97-104.

10. Jalini L, Lund J, Kurup V (2016) Nipple reconstruction using the C-V flap technique: long-term outcomes. Int J Surg Med 2(3): 162-166.

11. Zhong T, Antony A, Cordeiro P (2009) Surgical outcomes and nipple projection using the modified skate flap for nipple-areolar reconstruction in a series of 422 implant reconstructions. Ann Plast Surg 62(5): 591-595. 


\section{International Journal of Transplantation \& Plastic Surgery}

12. Nligan P (2013) Plastic surgery. London [U. A.]: Elsevier Saunders 5(1): 5.

13. Kim YE, Hong KY, Minn KW, Jin US (2016) A novel nipple reconstruction technique for maintaining nipple projection: the boomerang flap. Arch Plast Surg 43(5): 470-473.
14. Magno S, Terribile D, Franceschini G, Fabbri C, Chiesa F, et al. (2009) Accessory nipple reconstruction following a central quadrantectomy: a case report. Cases J 2: 32. 